\title{
eJRIEPS
}

Ejournal de la recherche sur l'intervention en éducation physique et sport

$30 \mid 2013$

Varia

\section{Modèles, analyse qualitative et configurations prototypiques dans les sports collectifs}

Models, qualitative analysis and prototypic configurations in team sports

Jean-Francis Gréhaigne, Patrick Marle et Zeineb Zerai

\section{(2) OpenEdition}

Journals

Édition électronique

URL : http://journals.openedition.org/ejrieps/2354

DOI : 10.4000/ejrieps.2354

ISSN : 2105-0821

Éditeur

ELLIADD

\section{Référence électronique}

Jean-Francis Gréhaigne, Patrick Marle et Zeineb Zerai, « Modèles, analyse qualitative et configurations prototypiques dans les sports collectifs », eJRIEPS [En ligne], 30 | 2013, mis en ligne le 01 juillet 2013, consulté le 04 octobre 2019. URL : http://journals.openedition.org/ejrieps/2354; DOI : 10.4000/ ejrieps.2354

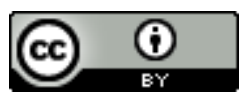

La revue eJRIEPS est mise à disposition selon les termes de la Creative Commons Attribution 4.0 International License. 
eJRIEPS 30 juillet 2013

Modèles, analyse qualitative et configurations prototypiques dans les sports collectifs

Jean-Francis Gréhaigne*, Patrick Marle* \& Zeineb Zerai**

* IUFM de Franche-Comté

** L'ISSEP Ksar Said Université la Manouba. Tunis, Tunisie

Résumé

L'ambition de ce texte est de revenir sur le concept de modèle du jeu et sur la notion de configuration prototypique que nous avons conçue et utilisée pour rendre compte d'une analyse qualitative du jeu en sport collectif. En effet, l'élaboration de théories ne saurait se concevoir aujourd'hui sans un recours à une présentation formalisée inspirée des modèles conceptuels. L'objectif principal d'un modèle conceptuel est de transmettre les principes fondamentaux et les fonctionnalités de base du système qu'il représente.

Il sera également envisagé quelques outils qualitatifs pour aider à l'analyse du jeu. Les décisions des joueurs ne sont pas que rationnelles au sens où elles sont aussi le fruit de l'interaction des autres composantes de l'action comme l'expérience des joueurs dans ses aspects conceptuels certes, mais aussi moteurs, sensibles, et sociaux.

Mots clés : modèle, configuration, décision, sport collectifs

L'ambition de ce texte est de revenir sur le concept de modèle du jeu et sur la notion de configuration prototypique que nous avons conçue et utilisée (Gréhaigne, 2007) pour rendre compte d'une analyse qualitative du jeu en sport collectif. En effet, l'élaboration de théories ne saurait se concevoir aujourd'hui sans un recours à une présentation formalisée inspirée des modèles conceptuels. L'objectif principal d'un modèle conceptuel est de transmettre les principes fondamentaux et les fonctionnalités de base du système qu'il représente. En outre, un modèle conceptuel doit être développé de manière à fournir une interprétation du système facile à comprendre pour les utilisateurs de modèles. Pour Mylopoulos (1992, p. 3) «La modélisation conceptuelle est l'activité de décrire formellement certains aspects du monde physique et social qui nous entoure à des fins de compréhension et de communication ». 


\section{eJRIEPS 30 juillet 2013}

Une modélisation conceptuelle adéquate doit donc proposer de nouvelles perspectives et un ensemble d'outils nouveaux pour promouvoir le progrès dans l'activité concernée. Un modèle conceptuel est un modèle qui existe seulement dans notre tête. Donc, ce terme peut être utilisé pour qualifier les modèles qui sont établis après un processus de conceptualisation. Ce mécanisme chez l'observateur ou le joueur représente l'effort de celui-ci pour penser et résoudre des problèmes posés par le jeu.

Néanmoins, dans la modélisation du jeu en sport collectif, la représentation de l'évolution du rapport de forces et de ses mécanismes est forcément en partie fausse ou incomplète du fait de la complexité du jeu et de données difficilement maîtrisables. Ce constat renvoie pourtant à une question essentielle : que signifie «modéliser » pour des chercheurs ?

Les sciences qui ont pour objet d'étude le jeu ne prétendent pas proposer un décalque de la réalité. Elles proposent plus simplement des modèles de représentations de cette réalité. Toutes les connaissances, toutes représentations conscientes référées à un objet, reposent à la fois sur des intuitions et des concepts. L'intuition est plutôt une représentation singulière, le concept est une représentation de portée plus générale. Ces représentations sont continuellement améliorées et précisées, mais aussi revisitées de manière critique par les scientifiques permettant ainsi de regrouper, dans un cadre théorique cohérent, l'ensemble des observations scientifiques du phénomène étudié.

L'idée de modélisation correspond à l'action de production de modèles. Un modèle peut être défini comme un système figuratif reproduisant en partie la réalité sous forme schématisée la rendant ainsi plus compréhensible. La réalité n'étant ni limpide ni directement accessible, il faut la décoder, la simplifier en ne conservant que les éléments et les relations qui semblent pertinents pour chaque sujet traité. Un modèle est une construction, une structure que l'on peut utiliser comme référence, une image analogique qui permet de matérialiser une idée, un concept, une action qui sont rendus ainsi plus directement assimilables et ainsi utilisables. Le modèle doit pouvoir symboliser un certain ensemble de faits à travers une structure explicative générale que l'on peut confronter à la réalité pour voir si elle reste pertinente. Plusieurs modélisations distinctes sont possibles à partir d'un même problème. Souvent, aucune n'est parfaite, au sens où elle ne rend compte que partiellement de toutes les données du problème. Cela donne lieu à des conflits entre les différents modèles scientifiques. La résolution des conflits s'opère alors identifiant de nouvelles connexions entre les données qui font évoluer le modèle même si des données restent non connectées entre elles. 


\section{eJRIEPS 30 juillet 2013}

\section{CONFIGURATIONS DU JEU}

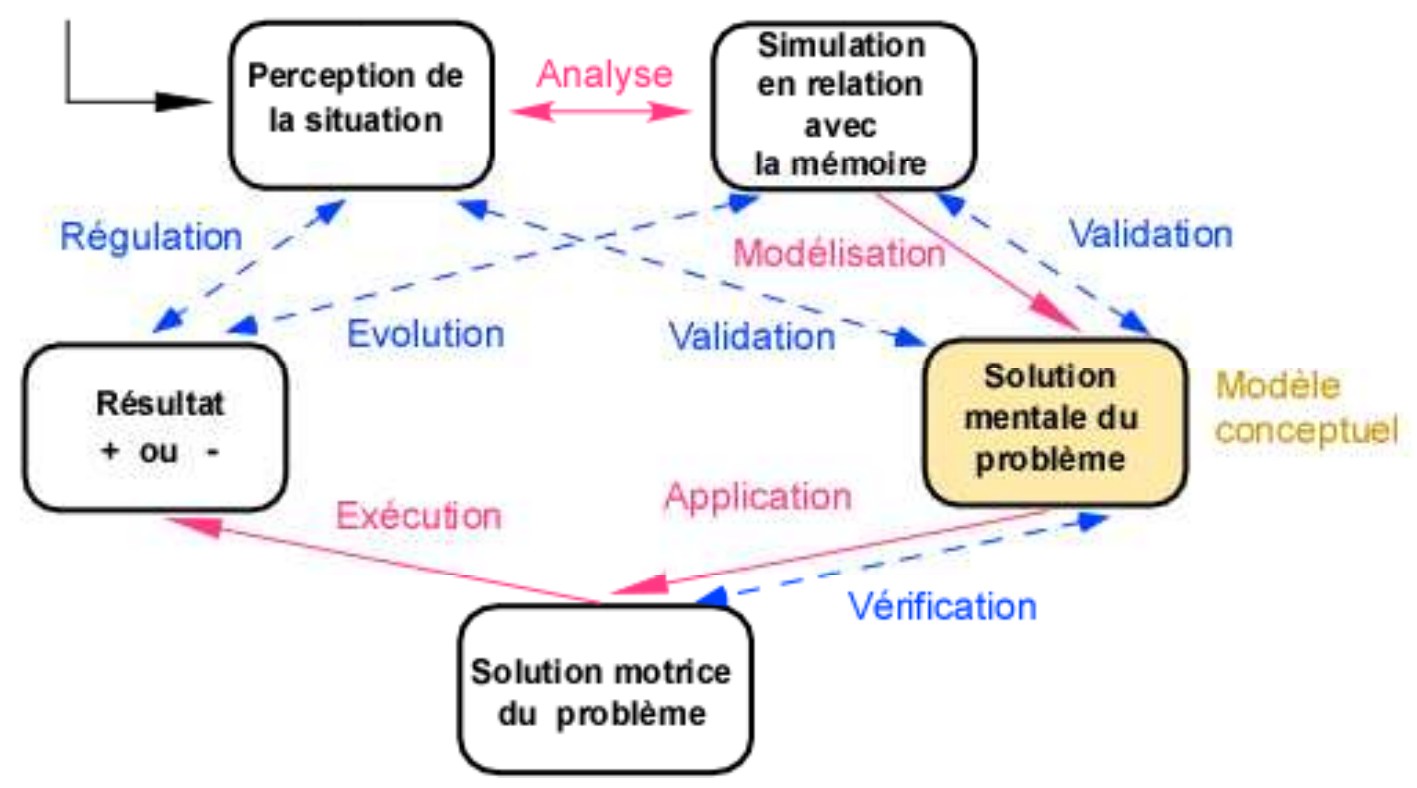

Figure 1. Le modèle revisité de l'action tactique (Malho, 1969).

Chaque dimension, si moderne et novatrice soit-elle, n'offre à elle seule qu'un pouvoir explicatif limité. La représentation fine et solide naît plutôt de la superposition, de la combinaison, de l'articulation de plusieurs grilles de lecture. Dès lors, quand et comment décide-t-on que la représentation d'un objet d'étude est bonne ? Que comprend-on d'un phénomène ? Comment l'évalue-t-on ? Pour nous, le fait qu'un modèle puisse aider à anticiper l'évolution des configurations du jeu est sans doute un bon indicateur de sa qualité. Si l'on reprend le modèle bien connu de Malho (1969) nous pouvons, à l'aide de divers processus, préciser les modalités de fonctionnement de ce modèle. II apparaît bien évident que le modèle conceptuel joue un rôle central dans le développement de la compréhension d'un système global (figure 1).

II est clair que si le modèle conceptuel n'est pas bien conçu, l'exécution de la solution motrice ne pourra pas être mise en œuvre correctement, provoquant des erreurs. Ici, pour éviter les maillons faibles dans la conception d'un système de décision tactique, il faudra être très minutieux avec les exigences et les obligations du jeu. L'objet de chacune des actions offensives est de provoquer, d'exploiter un déséquilibre du dispositif adverse, de créer l'effet de surprise, l'imprévisible, afin de marquer un but. Les attaquants doivent s'efforcer de prendre de vitesse la reconstitution par l'équipe opposée d'un équilibre défensif ou d'amener le barrage adverse dans une position critique et de rompre ainsi l'équilibre à leur avantage. Un exemple de déséquilibre en faveur de l'attaque est présenté figure 2 avec un attaquant en avance sur le repli défensif. 


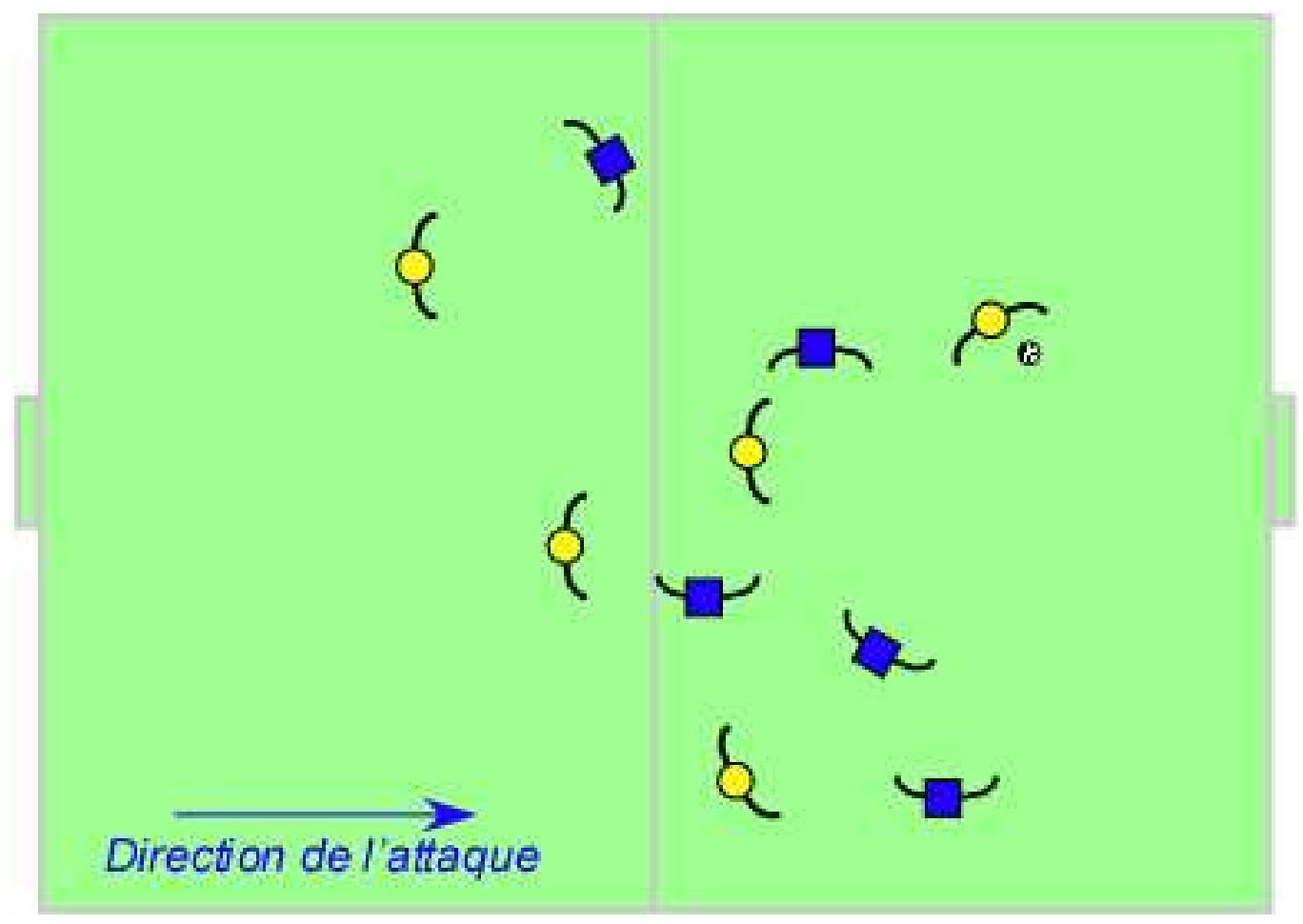

Figure 2. Exemple d'un porteur de la balle en avance sur le replacement défensif.

On le voit, si les systèmes complexes ne sont pas réductibles à des modèles explicatifs simples, ils nous sont toutefois intelligibles. Nous pouvons à chaque instant tenter de les modéliser, autrement dit élaborer et concevoir des constructions symboliques à l'aide desquelles nous pouvons raisonner des projets d'action au sein d'un système complexe, en anticipant consciemment leurs conséquences sur le jeu. Ces constructions symboliques sont en rapport très étroit avec l'observation voire l'évaluation des configurations momentanées du jeu en fonction des indices que les joueurs y prélèvent. L'utilisation du terme "symbolique " dans ce système de références ne renvoie pas à la notion habituelle et conventionnelle de symbolisme. II se réfère plutôt à une théorie assez spécifique des mécanismes centraux de l'organisation de la conscience : la perception, la connaissance, la mémoire et l'adaptation à une situation spécifique mais aussi, la mise en relation de ces différents mécanismes entre eux (cf. Frith, 2007 pour une revue). L'argument, en bref, est que la connaissance humaine est unique dans la mesure où l'on peut dire que c'est symbolique dans la fonction et dans le mode de fonctionnement.

Ces constructions symboliques reflètent un monde réel mais ce n'est pas la réalité. C'est un monde conceptuel indispensable pour créer du sens. Ce qui nourrit cette mémoire est constitué par toutes les informations qui ont été perçues par les différents sens, volontairement et/ou non volontairement. II s'agit donc d'informations qui peuvent être 


\section{eJRIEPS 30 juillet 2013}

récentes ou lointaines et qui ont été stockées. Ce contenu de la mémoire varie donc avec chaque joueur et ainsi nous pouvons dire qu'il y a autant de mémoire, de perception qu'il y a de joueurs constituant l'univers de chacun.

Mais on peut parler aussi de rationalité limitée c'est-à-dire une rationalité qui ne traite pas toutes les informations mais en privilégie certaines. Cela nous renvoie à l'idée d'image opérative (Ochanine, 1978) qui semble être la conséquence des limites de notre propre cognition. L'auteur souligne que l'information contenue dans l'image opérative est " structurée en un tout cohérent dont les constituants s'inscrivent dans un ensemble de relations déterminées " (Ochanine, 1969, p. 1). "L'évolution de l'image opérative au cours des apprentissages fait que les détails peu significatifs sont négligés au profit d'indicateurs plus adéquats " (Gréhaigne, 2011, p. 25). Trois caractéristiques essentielles façonnent l'image opérative : la finalisation, le laconisme et les déformations fonctionnelles (Ochanine, Quaas, \& Zaltzman, 1972).

La finalisation est la propriété principale de l'image opérative. En effet, Ochanine distingue l'image opérative de l'image cognitive du fait que la première se forme au cours d'une action donnée sur un objet, alors que la seconde est le reflet intégral de l'objet. Le fait que le joueur doit continuellement ajuster son image opérative aux objectifs poursuivis est une preuve de la finalité de cette construction mentale. Pour Ochanine, l'image opérative ne retient que ce qui est directement utile à l'action, en ce sens, elle est sélective. Tout doit être économique : par rapport à l'image cognitive, elle est laconique. Nous retrouvons ici l'idée que le reflet subjectif de l'objet construit dans la conscience du joueur n'est pas le reflet d'un miroir. Enfin, Ochanine va plus loin dans cette idée de sélection des informations pertinentes, pour lui l'image opérative est une déformation fonctionnelle de la réalité. La déformation fonctionnelle est l'accentuation des informations les plus importantes en fonction de la tâche visée : propriétés de l'objet, ses divers aspects, ses structures partielles. Cette déformation est dite fonctionnelle tout d'abord parce qu'elle implique que le reflet soit toujours adéquat à la réalité. Pour Ochanine, la déformation fonctionnelle des images opératives vise toujours à supprimer ou à ramener au minimum l'incertitude de l'action en cours. Enfin, la déformation est fonctionnelle car elle manifeste la souplesse et la plasticité de l'image opérative.

On peut ajouter, ici, que tout apprentissage nécessite une plasticité neuronale (Paillard, 1987). Plus une information est présente dans le jeu, plus notre cerveau est sollicité. Ainsi, par la répétition, plus ou moins fréquente, nous sélectionnons et renforçons les liaisons 


\section{eJRIEPS 30 juillet 2013}

entre les zones neuronales; il sera alors plus facile de se remémorer rapidement une configuration du jeu ou une information.

\section{La réalité du jeu et son appréhension}

Une situation de jeu évolue d'un état 1 à un état $n$. Une configuration de jeu définit la position des joueurs à un instant t. Dans l'analyse des choix tactiques des différents joueurs lors d'un mouvement collectif, nous voulions obtenir des "états dynamiques" des joueurs qui participent à l'attaque ainsi que ceux de leurs adversaires. Mais le problème n'est pas simple à résoudre. Effectivement, la transmission d'un événement à travers un système d'interfaces est forcément incomplète du fait de la perte d'informations ; elle aboutit nécessairement à une configuration subjective. II en résulte une perte considérable dans la transmission de l'information mais ce phénomène est paradoxalement positif. C'est, en effet, l'indétermination partielle des structures qui est source de transformation. Le gain en plasticité, souplesse, aisance d'adaptation, possibilité de progrès et de développement..., compense de beaucoup ce qui est perdu en transmission stricte d'informations.

Toute interprétation cognitive devient alors une sorte d'enjeu, de pari au cours duquel le joueur introduit une détermination subjective pour combler les indéterminations liées à la plasticité. A l'inverse, c'est parce que cette détermination subjective est en partie erronée qu'une correction ultérieure est possible. En définitive, la notion de plasticité donne toute leur signification aux notions "d'autonomie" et de "cohérence", car toute connaissance construite est fondamentalement personnelle et incluse dans l'ensemble partiellement indéterminé des conduites du sujet. II permet la construction de structures malléables au sein desquelles la cohérence ne va pas de soi, n'est jamais aboutie mais doit être recherchée activement. Le concept central, sous-jacent à cette conception est celui d'adaptation de l'entraîneur ou du joueur. « L'apprentissage perceptif consiste à extraire des schèmes de configurations d'indices pertinents caractéristiques dont la covariance ou la co-présence, dans une situation donnée, permet de réduire le temps d'analyse et d'évolution du contexte informationnel, par le choix d'indicateurs privilégiés, prédictifs de la situation globale » (Paillard, 1987, p. 1422). Dans notre cas, ces configurations d'indices sont fournies par l'activité du joueur face à des configurations du jeu évolutives. Cette activité du joueur confère progressivement une significativité particulière à certains événements du jeu. Pour un joueur expert, ces événements dans une succession de situations ont été extraits pour constituer un cadre de références permettant d'anticiper la 


\section{eJRIEPS 30 juillet 2013}

probabilité d'apparition de certaines difficultés, de certains comportements ou attitudes des élèves.

Sur le plan épistémologique, ce sont ces configurations d'indices en relation avec la plasticité qui permettent qu'une connaissance soit efficace alors qu'elle n'est pas isomorphe à l'événement auquel elle correspond. C'est la plasticité qui explique qu'une connaissance soit toujours provisoire, constamment ouverte sur une révision et/ou un progrès. Elle favorise l'acquisition rapide de connaissances approximatives tout en maintenant ouvertes des possibilités de révision ultérieure. Ainsi, le flou cognitif, loin d'être un élément péjoratif, est la condition et peut être la cause même de la connaissance apprise. On voit alors que le fonctionnement du sujet renvoie souvent à « l'à peu près ", au flou cognitif et pourtant ce sujet prend des décisions, agit... En fonction du degré d'adéquation des réponses par rapport à l'effet recherché, il y a soit modification des moyens mis en oeuvre pour réduire les écarts avec les effets attendus, soit stabilisation des réponses, soit complexification de la situation en vue d'amener l'élève à un niveau supérieur de pratique. Le gain apporté par cette généralisation des apprentissages conduit les joueurs à des réponses plus rapides et plus simples. II correspond, dans un premier temps, à des processus profondément personnels car la mise en relation des éléments qui ont permis la réussite possède comme principales caractéristiques d'être singuliers et individuels. Ensuite, c'est la mise en commun et le choix entre des solutions différentes qui permet le progrès, cette procédure évoquant tout à fait la notion "d'alternances fonctionnelles » chère à Wallon (1941). Ainsi se construit l'expérience du joueur expert avec une succession de processus internes et externes.

Pour bien clarifier notre propos, nous allons avec Mouchet (2005) tenter de différencier expert et expérimenté. De manière générale l'expérience est souvent considérée comme construction personnelle, progressive et lente. Dewey (1947) souligne que l'expérience est une transaction avec l'environnement naturel et humain et qu'elle s'organise en se développant selon les deux principes de continuité, principe longitudinal de l'expérience qui l'intègre dans le développement et l'interaction, principe latéral de l'expérience qui la coordonne à d'autres expériences ce qui la distingue d'un simple vécu.

Ainsi, l'expérience est faite de la succession d'expériences et de leur continuité, chacune affectant la qualité de l'expérience future. Elle est donc à distinguer de l'expertise qui s'appuie sur elle sans s'y résumer ; "nous caractérisons plutôt l'expertise, par le niveau de compétition des joueurs » (Mouchet, ibid). Un joueur peut donc être expert sans avoir beaucoup d'expérience et un sujet expérimenté n'est pas forcément un expert. Plus 


\section{eJRIEPS 30 juillet 2013}

précisément, Mialaret (1998, p. 165) définit l'expérience comme « un ensemble d'informations, de connaissances, d'attitudes acquises par un individu au cours de son existence par l'observation spontanée de la réalité et de ses pratiques, le tout intégré progressivement à sa personnalité ». Cette expérience acquise de façon presque non consciente varie d'un sujet à l'autre, dépend de la personnalité du joueur et s'inscrit dans les comportements, les habitudes, les façons de penser et de faire (cf. Bourdieu, 1980 avec le concept d'habitus). Enfin Leplat (2000) estime que ces sortes d'habitus sont à la fois des instruments de lecture de la réalité et des producteurs de pratiques qui répondent à cette lecture. L'analyse des logiques intrinsèques de chaque joueur, révèle ainsi une mobilisation singulière et circonstanciée des repères communs externes.

Dans les sports d'équipe, les joueurs ont élaboré des capacités d'analyse qui fonctionnent très rapidement. Par exemple, les joueurs de haut niveau ont développé la capacité de reconnaître rapidement et de mémoriser les configurations du jeu exécutées par leurs adversaires. Cette capacité ne repose pas sur le fait que les joueurs de haut niveau ont une plus grande mémoire mais sur le fait que leur mémoire spécifique appliquée aux sports de stratégie est tout simplement plus détaillée et plus sélective que pour le commun des mortels. Elle se développe par le biais d'années de pratique, d'expérience et la construction d'outils spécifiques (Bouthier, 1997 ; Ripoll, Zoudji, \& Lluccia, 2009).

\section{Des outils qualitatifs pour l'analyse du jeu}

La notion centrale d'opposition nous a amenés à considérer les deux équipes impliquées dans un match comme l'interaction des systèmes organisés (Gréhaigne, Godbout \& Zerai, 2010). On peut alors concevoir qu'une analyse systémique d'un sport d'équipe est fondamentale pour l'apparition d'une nouvelle compréhension du jeu. Plus généralement, dans une approche classique de l'apprentissage, on enseigne d'abord aux étudiants les compétences techniques du jeu puis on envisage leur utilisation en jeu.

Nous sommes tentés de dire que ce qui est plus important, c'est de faire en sorte que les joueurs soient capables de gérer d'une façon optimale le désordre (Gréhaigne, 1989; Gréhaigne \& Godbout, 1995; Villepreux, 1987). Un jeu repose rarement sur l'application simple de combinaisons tactiques apprises précédemment pendant l'entraînement. Ainsi, la plupart du temps pendant le jeu, on ne peut prévoir que des probabilités d'évolution pour les configurations d'attaque et de défense, d'où l'importance de connaissances tactiques) pour un joueur afin de résoudre les problèmes posés par le jeu et ainsi jouer plus rapidement et plus efficacement que l'adversaire. 


\section{eJRIEPS 30 juillet 2013}

Cette approche de l'entraînement qui met en avant «l'opposition » et «la gestion du désordre ", comme base pour n'importe quel progrès, met en lumière les nouveaux concepts qui apparaissent fondamentaux pour un renouvellement de l'entraînement, dans les sports d'équipe. Bouthier (1986), Bunker \& Thorpe (1982), Deleplace (1979), Gréhaigne (1992) et Turner \& Martinek (1992) ont montré avec des études différentes la nécessité de passer d'une approche de technique à une approche basée sur la compréhension. En effet, il ne faut pas oublier qu'à cette époque les entraînements étaient souvent très technicistes, très pauvres en terme de tactique et essentiellement basés sur la répétition.

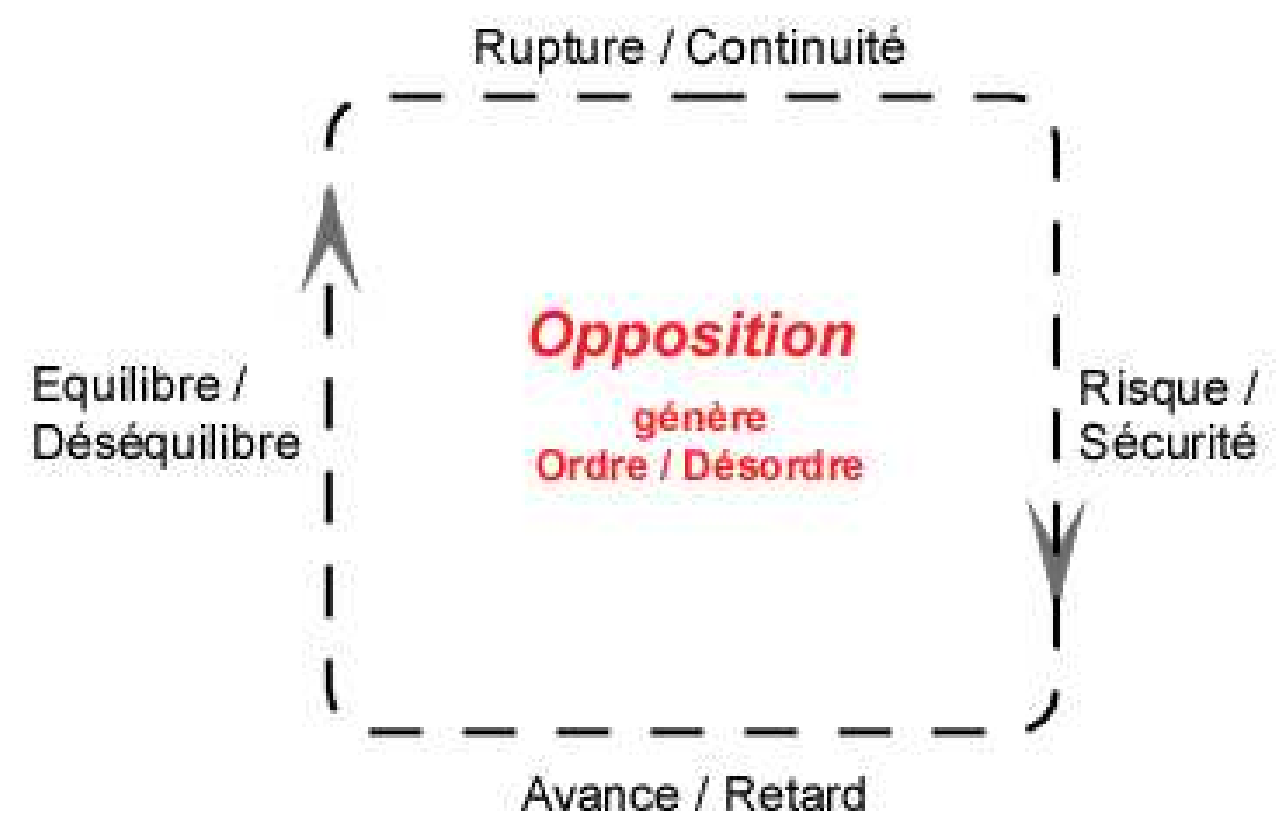

Figure 3. Concepts généraux liés à la notion d'opposition (adapté de Gréhaigne, 1992)

La figure 3 identifie quelques concepts dialectiques qui deviennent centraux quand on conçoit l'opposition comme un tout indissociable et comme un élément incontournable de l'étude des configurations du jeu. Ces concepts généraux peuvent être complétés par des critères qualitatifs d'analyse des configurations momentanées du jeu en vue de la constitution d'une base de données spécifique de l'affrontement. Ainsi cette base différera des données habituelles constituées par le score, les profils des joueurs, l'analyse des matchs, les statistiques à propos du jeu.

L'évolution de la dynamique du jeu (figure 4) ne peut alors bien se modéliser qu'en concevant une évolution discontinue dans le temps. II nous semble qu'une base de données pourrait être constituée à partir de la mise en relation de cinq critères :

- position sur le terrain et volume de l'espace de jeu effectif (EJE) ;

- position et circulation du ballon ; 


\section{eJRIEPS 30 juillet 2013}

- positions respectives de l'espace de jeu effectif offensif (EJEO) et de l'espace de jeu effectif défensif (EJED) ainsi que leurs dynamiques d'évolution ;

- défense en barrage ou à la poursuite ;

- l'extension ou la compression de l'EJE dans plusieurs configurations, permet d'obtenir une représentation immédiate et assez exacte du rapport d'opposition.

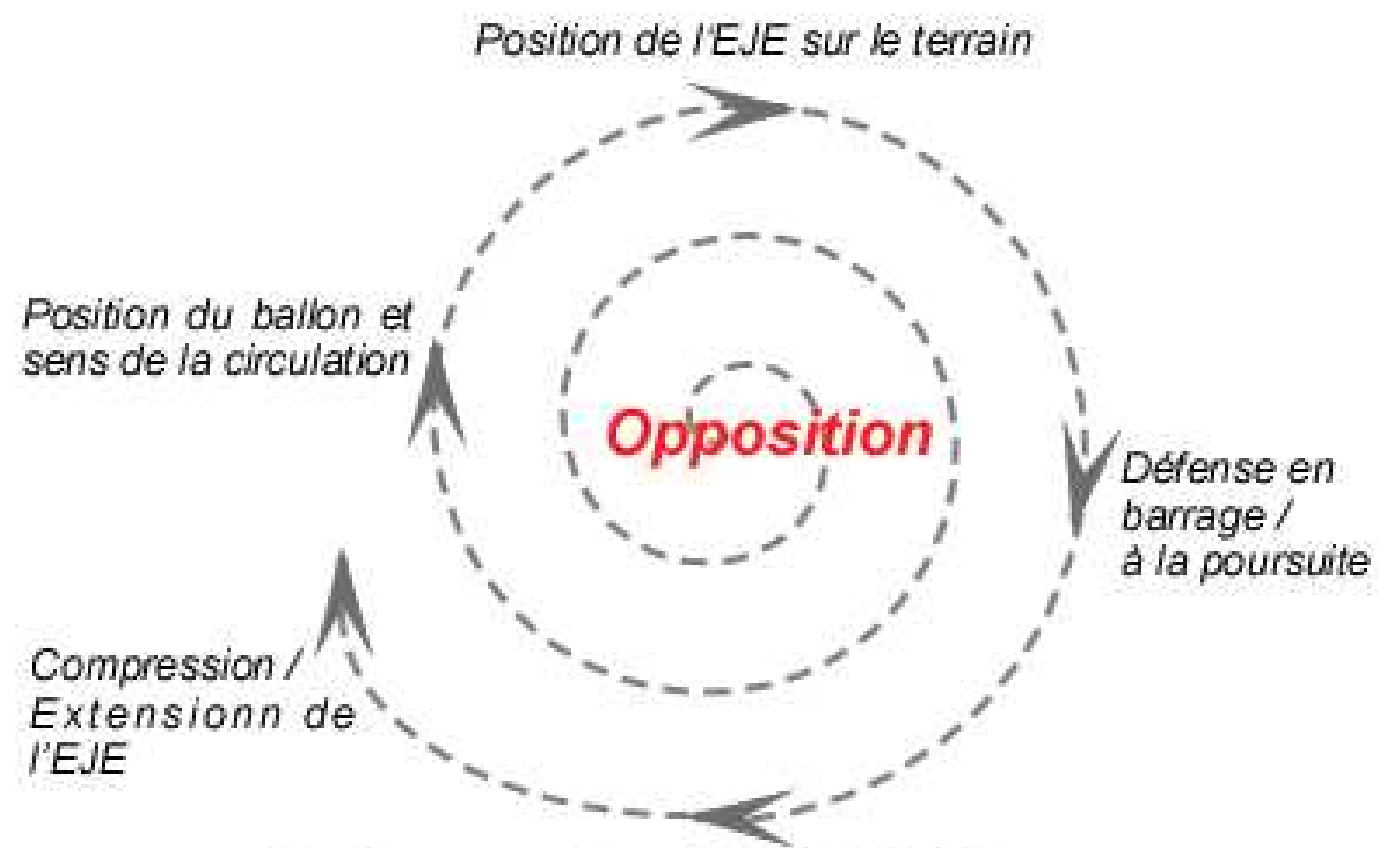

Positions respectives de EJEO et EJED

Figure 4. Critères d'analyse d'une configuration momentanée du jeu.

Avec la figure 5, nous allons illustrer l'utilisation de ces critères d'analyse avec une configuration du jeu donnée. L'espace de jeu effectif est situé à cet instant au milieu du terrain. Le ballon est à l'arrière de l'espace de jeu effectif à la périphérie et nous sommes dans une phase de montée de la balle par les ronds jaunes avec une défense des carrés bleus qui est en barrage. L'EJEO et EJED sont peu interpénétrés. Dans cette sorte de photographie, l'EJED est en contraction tandis que l'EJEO est plutôt en expansion. Au football dans les jeux réduits, c'est une configuration assez classique qui offre une certaine «stabilité » momentanée du jeu. Néanmoins, à l'interface de l'attaque et la défense la situation à double effet (Delaplace, 1966) lie organiquement offensive et défensive en soulignant l'immédiateté du passage d'attaquant à défenseur. Cette organisation à double effet vise, quand on est en défense, à prévoir déjà un ou des schémas de jeu qui permettent de lancer la contre-attaque dès la récupération du ballon à l'aide de joueurs placés en avant. Si l'on est en attaque, les joueurs placés en soutien, 


\section{eJRIEPS 30 juillet 2013}

c'est-à-dire en arrière du porteur de balle doivent envisager dans leurs actions qu'ils constituent, aussi, la première ligne défensive en cas de perte de balle.

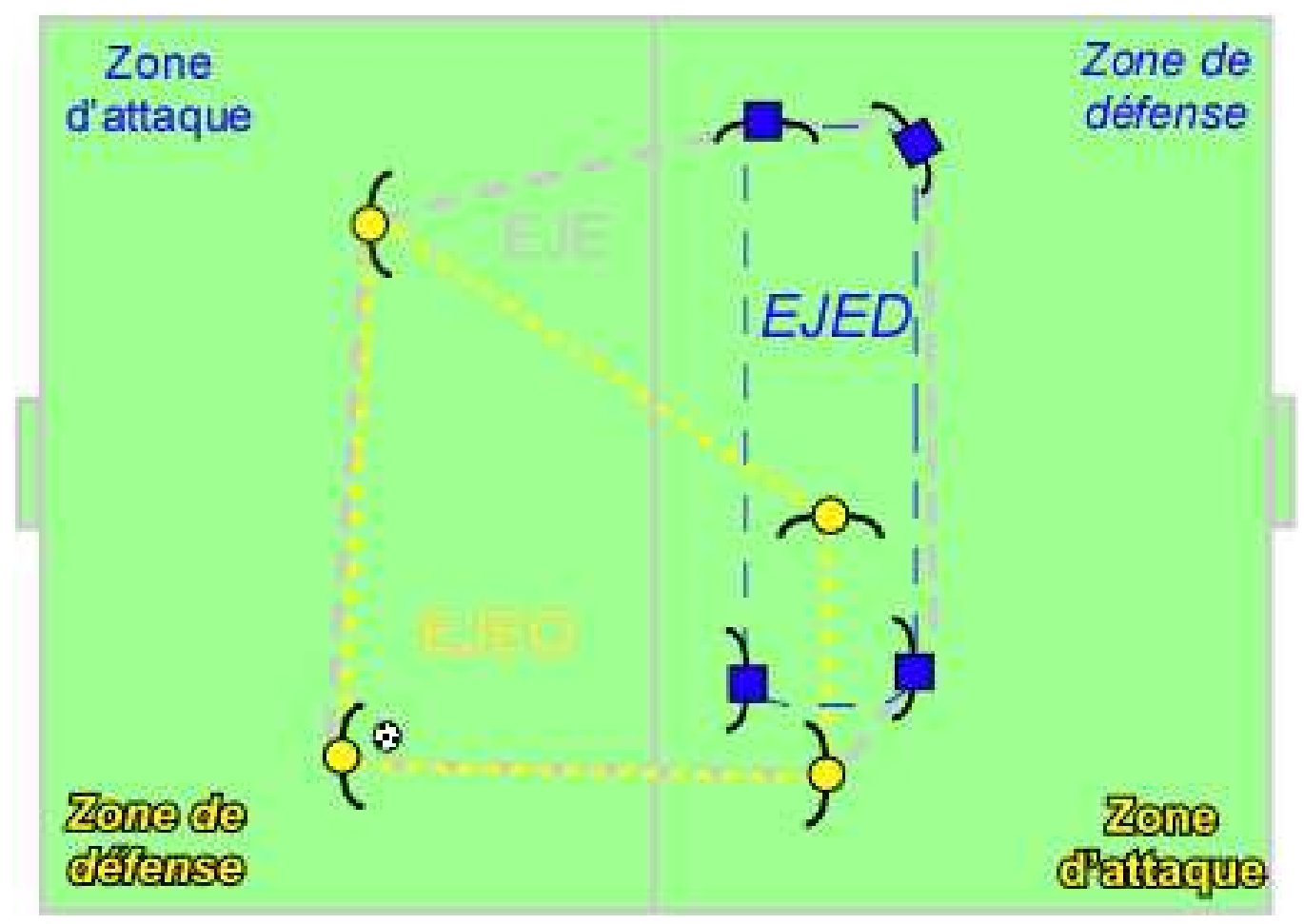

Figure 5. Exemple de configuration du jeu.

Dans ce deuxième exemple (Fig. 5), le déséquilibre est à construire pour les attaquants. Cette configuration présente aussi quelques dangers potentiels pour ceux-ci en cas de récupération de la balle à l'arrière de l'EJE. En effet, un défenseur peut monter très vite sur le porteur de balle afin de provoquer un $1 \times 1$ dont l'issue n'est pas évidente car les possibilités de passes deviennent restreintes. Si la balle est récupérée, le chemin du but est ouvert et une contre-attaque rapide et très dangereuse peut être mise en place.

Concernant l'étude de la dynamique de ce rapport d'opposition à un niveau plus macroscopique, l'opposition, la continuité et la réversibilité sont trois autres concepts qui s'avèrent également essentiels.

- L'opposition renvoie à l'affrontement des deux groupes (représenté par l'EJEO et l'EJED) dans un système dynamique spatialement défini par une aire de jeu et un temps limité dans un match. Elle repose fondamentalement sur des états d'équilibre et de déséquilibre quand le rapport de forces entre les deux équipes n'est pas trop déséquilibré.

- La continuité du jeu et le jeu en mouvement engendrent du désordre provoquant des désorganisations qui tendent à être compensées tant que la défense peut s'adapter. 


\section{eJRIEPS 30 juillet 2013}

Bien souvent, ce désordre est un désordre apparent où l'on a tout lieu de supposer qu'il existe une forme d'ordre caché à qui sait le décoder (circulation des joueurs et du ballon).

- Enfin, la réversibilité se produit quand la perturbation n'a pas pu être régulée par les attaquants, entraînant la perte de la balle. La réversibilité des situations représente un aspect fondamental des sports collectifs en rapport avec le fait que les équipes attaquent ou défendent à tour de rôle. Cette réversibilité est à considérer dans un rapport dialectique continuité / rupture.

Dans le jeu en mouvement, au principe de circulation dans les lignes défensives de la matrice défensive correspond un principe de transformation des mouvements dans la matrice offensive. De ce point de vue, les matrices offensives et défensives sont basées sur des principes d'organisation dynamique. L'exécution se construit en fonction des données fournies par la partie d'orientation de l'action, elle rationalise cette dernière. Cela permet d'expliquer en partie l'apparente difficulté des joueurs à décrire leurs actions et le pourquoi des décisions prises. La vitesse du jeu y est peut-être pour quelque chose mais plus sûrement quand une partie de la base de données s'est condensée et automatisée, alors elle est moins présente dans la conscience du joueur sauf alerte.

Nous pouvons maintenant proposer avec une grande partie de ces données un modèle (figure 6) qui intègre le mouvement. Ainsi, ce modèle dynamique fonctionne dans un espace fini, le terrain, ce qui restreint le champ d'expansion. Une des propriétés de l'affrontement est de présenter une certaine élasticité. Dans le jeu, ce système élastique est traversé par une succession de contractions et d'expansions. Pour les joueurs, il est nécessaire de stabiliser des états d'équilibre précaires donnant un peu de temps pour décider alors qu'une tension est appliquée au système attaque / défense. 


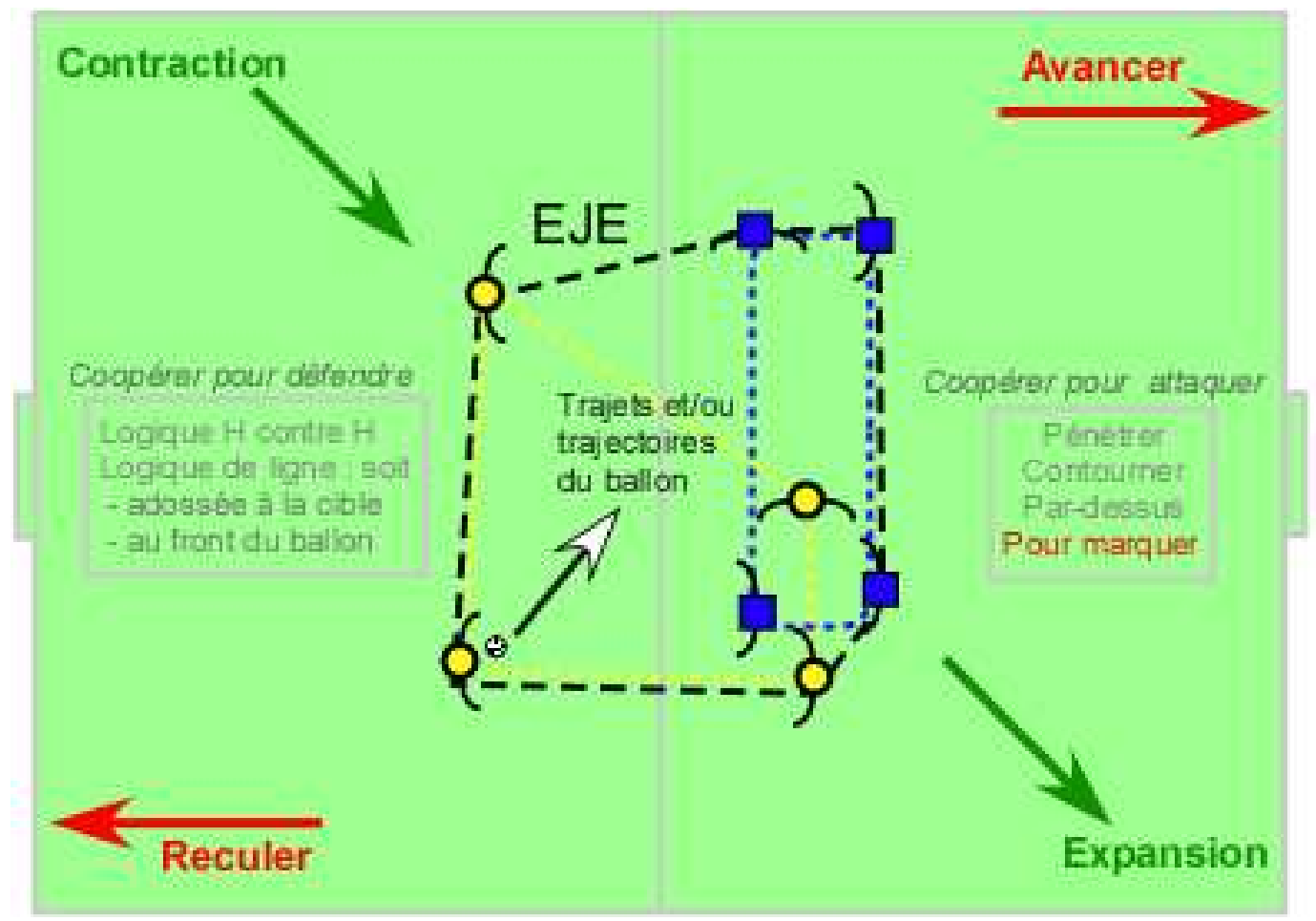

Figure 6. Un modèle dynamique d'analyse du jeu (Gréhaigne \& Godbout, 2012).

L'élasticité avec son énergie potentielle ouvre de nouvelles opportunités pour l'optimisation du jeu en permettant, à l'aide des processus cognitifs, de nouvelles transformations qui ne peuvent pas être appliquées aux systèmes trop rigides. Les systèmes élastiques fournissent une certaine tolérance aux retards dans les communications et les décisions. L'énergie élastique dans une configuration du jeu est constituée par une énergie statique (la position des joueurs) et une énergie dynamique (le mouvement en cours). Au cours du temps, cette énergie évolue principalement avec la variation des déplacements et des distances entre les joueurs. Le comportement d'un système défensif, quand il a été dévié d'une position d'équilibre par une perturbation, est une indication de la stabilité ou de l'instabilité du rapport d'opposition. Si le système défensif dévie de l'équilibre puis retourne rapidement à sa position initiale après que le facteur qui a causé la déviation ait été supprimé, on peut considérer que l'équilibre est stable. Si la déviation ne disparaît pas, mais continue à augmenter, l'équilibre est instable et l'attaque est, temporairement, dans une configuration favorable. La contraction ou l'expansion sous laquelle l'équilibre stable devient instable est appelée la charge critique pour le système. Si celle-ci persiste la défense demeure dans une position critique, en effet un barrage de joueurs, continu et pas trop étiré dans la largeur et la profondeur semble une position correcte pour la défensive. La stabilité d'un système élastique est donc la propriété de ce système à dévier 


\section{eJRIEPS 30 juillet 2013}

peu d'une position d'équilibre. L'établissement d'un répertoire d'états critiques constitue le problème principal de l'étude de la stabilité de systèmes défense / attaque afin de décrire le mieux possible les affrontements auxquels les joueurs risquent d'être confrontés pour les rendre intelligibles et prévisibles.

En mécanique, un exemple d'élastique générique peut être illustré par un ressort enroulé. Mais, dans le cas d'une configuration du jeu, l'élasticité n'est pas purement mécanique car les joueurs prennent des décisions qui peuvent perturber la contraction et l'expansion du système attaque / défense. Le mouvement des joueurs et du ballon produit des facteurs de déséquilibre et ici il faut aussi intégrer l'intentionnalité des joueurs qui « jouent » le jeu et du jeu. Cela signifie en particulier que dans la résolution des contextes où les décisions prédominent que ceux-ci doivent intégrer les variations possibles d'intentionnalité des joueurs : par exemple, le choix de «pénétrer », «contourner » ou «jouer par-dessus » pour les attaquants. Ces facteurs entraînent des fluctuations, des écarts, des déformations de la forme géométrique instantanée de la configuration du jeu. Cette élasticité peut, aussi, concerner le ballon : en fonction de la quantité d'énergie mise en oeuvre pour le propulser, cela induira un déplacement plus ou moins important de la balle.

Il est à noter que l'expression de stabilité des systèmes déformables est parfois utilisée en sport collectif. Le plus souvent, elle renvoie aux schémas tactiques qui sont des dispositifs établis à l'avance dans lesquels les joueurs et le ballon circulent et agissent de façon stéréotypée, conformément aux indications répétées à l'entraînement. Dans le jeu, le concept de stabilité de systèmes élastiques est étroitement connecté avec le concept de mouvement. L'énergie potentielle élastique produit des forces qui traversent et déforment la configuration momentanée du jeu faisant, ainsi, évoluer celui-ci de façon variée. Toutefois, certaines configurations reviennent périodiquement.

\section{Des configurations prototypiques}

C'est vrai, il n'y a pas de consensus sur ce qui constitue un «prototype »; le mot est souvent utilisé de façon interchangeable avec le mot «modèle» et cela peut prêter à confusion. Dans l'industrie, un prototype est souvent utilisé pour tester certains aspects d'une conception prévue d'un objet, sans tenter de simuler exactement la totalité des choix possibles. Dans les sports collectifs en testant différentes configurations momentanées du jeu, on vise, en premier au travers de prototypes, à identifier le fonctionnement du jeu. Dans l'équipe ces modèles sont destinés à aider à la prise de décision interne et constituent une partie du fond de jeu qui permet la compréhension par 


\section{eJRIEPS 30 juillet 2013}

chacun de l'évolution des rapports d'opposition. L'expérience qui consiste souvent à confronter un ou des joueurs à un prototype est principalement utilisée pour comprendre les décisions de ceux-ci. Aussi, ce type de modèle peut permettre une évaluation précoce de la façon dont un joueur interagit avec les éléments du champ de jeu, en particulier les partenaires en regard des différentes actions dans un rapport de forces donné. Avec ces premiers éléments, nous allons illustrer une configuration momentanée du jeu parmi les plus habituelles que l'on retrouve systématiquement dans le jeu des débutants. Nous avons dénommé ce type de configurations "prototypiques " au sens où il représente un modèle original, archétype d'un modèle qui se reproduit. L'étude de l'ensemble des configurations prototypiques devrait permettre aux élèves de construire des prototypes (cf. images opératives) par catégorisation de formes géométriques, de classes de propriétés d'objets et enfin des catégorisations de relations temporelles en vue d'être plus efficaces en jeu. On vise, ici, la mise à jour d'invariants pour reconnaître et décider vite à propos des configurations momentanées du jeu (Gréhaigne, 2007).

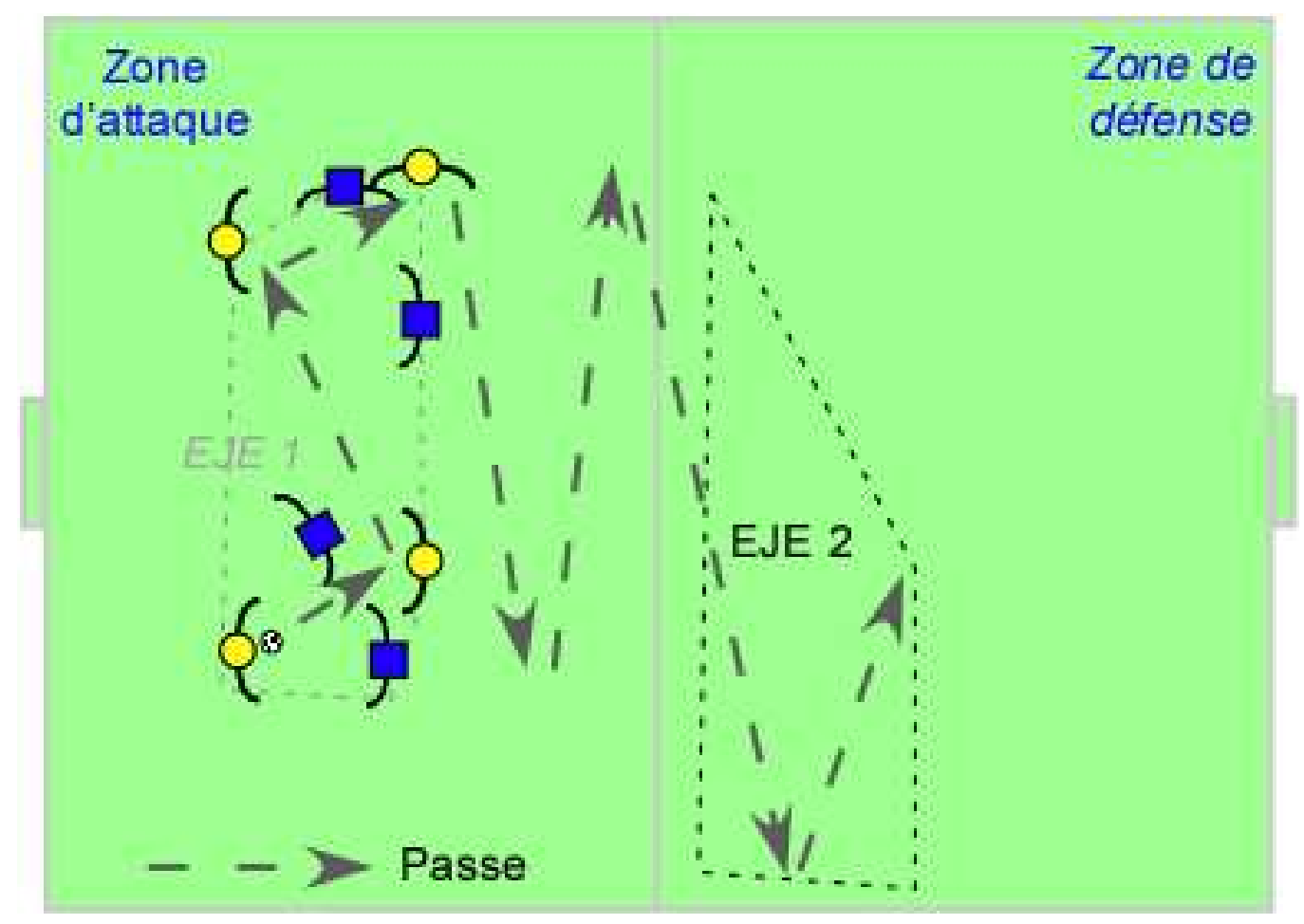

Figure 7. Une circulation de la balle à plat jusqu'à la perte du ballon.

La figure 7 illustre une circulation de la balle caractéristique des débutants quand le lancer long n'est pas encore bien construit. Après une mise en jeu sur la ligne de but, les attaquants s'échangent le ballon à l'aide de passes courtes en cloche en travers car la défense occupe l'axe central ou le devant du porteur de balle l'empêche de passer en 


\section{eJRIEPS 30 juillet 2013}

avant. Cette circulation de la balle à plat dans la dimension forte de la défense entraîne très fréquemment une perte du ballon au profit de l'équipe adverse et se traduit très souvent par un tir spontané du récupérateur.

Après la construction de la passe longue et de sa réception, les progrès sont liés au degré de compréhension du jeu par les joueurs. En effet, la relation entre le niveau de la pratique et le niveau de connaissances tactiques se vérifie pour les sujets déjà pratiquants d'un sport collectif à l'école. II semble que l'expérience a un rôle majeur dans la construction de connaissances tactiques et dans la variété des configurations prototypiques utilisées (Gréhaigne, Zerai, \& Caty, 2009 ; Zerai, 2009). Cette formalisation présente quelques risques et limites. Chaque solution constitue un peu une entité propre à un contexte toujours singulier. Les solutions formelles capables de résoudre un problème posé par le jeu peuvent être nombreuses, ce qui amène parfois l'enseignant à pratiquer des choix plus ou moins arbitraires. Ensuite, il faut admettre que toute modélisation représente comme nous venons de le voir un nécessaire appauvrissement de la grande variété des données, d'où la tentation de réduire les propriétés du jeu à quelques préceptes. Enfin de nombreux phénomènes liés à la communication et à la tactique résistent aux tentatives de formalisation.

Dans les sports collectifs où l'on tient le ballon (handball ou basket-ball par exemple), des caractères particuliers apparaissent car le jeu sur espace stabilisé prend relativement de l'importance. Dans ce cas, le perfectionnement chez les élèves passe souvent par la construction d'un code de la circulation des joueurs et de la balle. II s'agira donc de fournir ou de faire construire aux élèves des modèles du jeu à partir de quelques configurations prototypiques relativement stables. Leur appropriation suppose que les modèles proposés puissent évoluer en fonction des progrès, être discutés pour permettre une bonne stabilisation des apprentissages et enfin qu'on puisse les faire fonctionner pour prendre conscience de leur champ d'application et de leur validité. Ainsi, on pourra les rectifier et / ou les complexifier en y intégrant les conséquences des expériences nouvellement réalisées.

Après ce travail d'identification se pose maintenant le problème central de leur enseignement aux élèves. Après quelques expérimentations, il semble bien que la vision et l'étude par des élèves de configurations prototypiques du jeu bien choisies, présentées aux joueurs sous forme de vidéos, facilite l'apprentissage du jeu (voir Berchebru, 2007 pour une étude exhaustive de ces problèmes). Comme nous l'avons vu dans le premier paragraphe, la distinction faite par Ochanine (1978) entre images cognitives et images 


\section{eJRIEPS 30 juillet 2013}

opératives établit un fait essentiel : les joueurs qui veulent progresser dans le jeu doivent sélectionner les informations en vue de se forger une représentation la plus rentable possible.

Pour l'enseignant ou l'entraîneur, le recours fréquent aux mêmes configurations prototypiques constitue des repères de niveau de jeu quand elles apparaissent régulièrement dans une rencontre d'un rapport de forces donné. Cela autorise la mise en place d'une transversalité partielle dans la didactique des sports collectifs en aidant à l'utilisation, à partir de l'analogie, d'une même configuration prototypique dans deux sports collectifs différents. Par exemple, il est possible, avec des joueurs débrouillés, en cas d'échec de l'action rapide vers le but, qu'un jeu balle « en avant " apparaisse, c'est-à-dire que la balle recule momentanément pour faciliter la montée de balle.

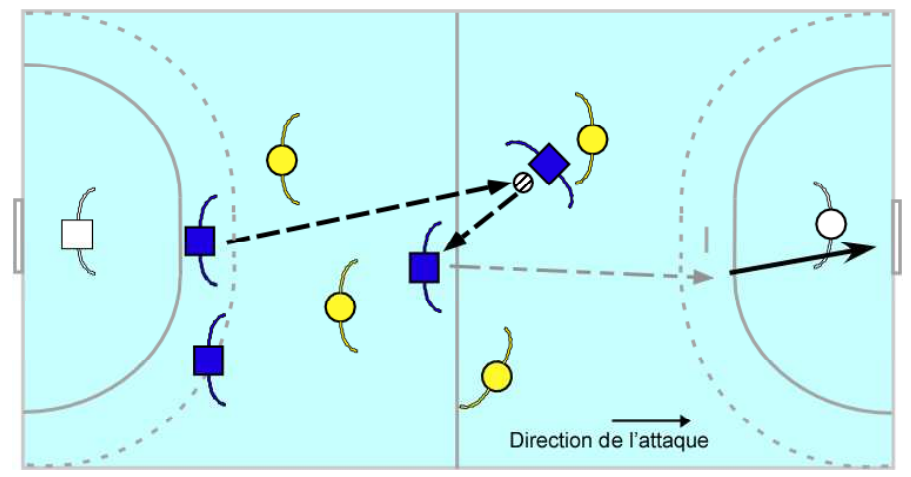

Figure 8. Un jeu balle "en avant" en handball.

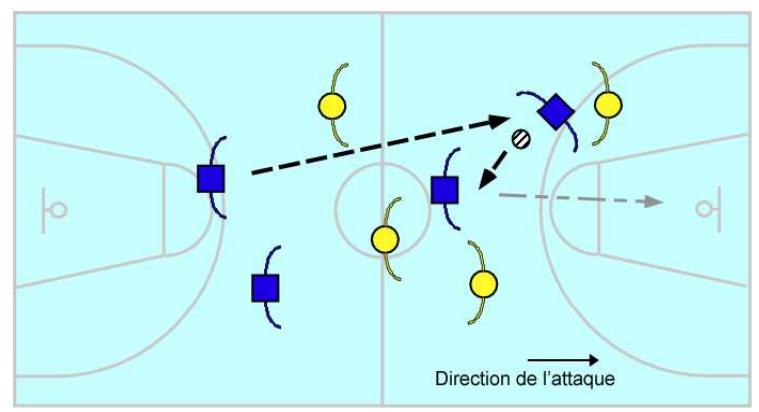

Figure 9. Un jeu balle "en avant" classique en basket-ball.

La figure 8 illustre le cas typique de montée de balle en handball avec une longue passe pour un joueur situé en avant qui étant bloqué par un défenseur passe la balle à un joueur en soutien qui dispose lui d'un espace ouvert devant lui. La suite de l'attaque est constituée par une conduite de balle en dribbles suivis d'un tir au but. La figure 9 décrit le même cas caractéristique d'un jeu balle « en avant » en basket-ball (cf. Zerai, Gréhaigne \& Godbout, 2011). Le recours à un soutien avec un partenaire qui continue l'attaque dans ces deux sports collectifs constitue bien une tactique identique. Aussi, attirer l'attention des joueurs sur certaines configurations prototypiques peut être utile avant l'action dans la façon de comprendre, d'organiser, de se préparer afin de répondre rapidement aux difficultés proposées par les rapports d'opposition.

Les ressemblances perçues entre deux ou plusieurs configurations du jeu permettent d'organiser les objets et les événements dans des catégories familières. Le processus de comprendre une nouvelle situation de jeu doit être conçu en terme de rapport avec ce qui 


\section{eJRIEPS 30 juillet 2013}

est déjà familier. Dans les sports d'équipe, les configurations prototypiques de jeu fournissent une sorte de modèle pour faire des inférences à partir de situations de jeu familières. Au cours de ce raisonnement par analogie, la nouvelle situation peut être rapprochée d'une configuration prototypique bien connue. Alors, l'analogie entre deux situations de jeu peut fournir des systèmes de référence pour apprendre à traiter promptement les configurations du jeu (Zerai, Gréhaigne, \& Godbout, 2013). En cas de réussite, le joueur se sera livré à une autoévaluation correcte de la situation permettant une bonne anticipation. Le recours au comparateur que constitue la configuration prototypique marque l'évolution de la capacité du joueur à réfléchir, prévoir et réguler ses propres actions. Toute situation ou débat permettant aux joueurs de s'appuyer sur des observations pour pouvoir apprécier l'adéquation entre anticipation et résultat obtenu constitue un point important pour les apprentissages. Bien entendu, dans ce processus, il s'agira de tenir compte du niveau initial des élèves et de veiller à ce que les problèmes posés par le jeu soient suffisamment nouveaux pour qu'ils soient intéressants mais aussi suffisamment voisins de configurations déjà maîtrisées pour que la résolution des problèmes soit possible.

\section{Conclusion}

L'ambition de cet article était de clarifier la notion de modèle en sport collectif et d'envisager quelques outils qualitatifs pour aider à l'analyse du jeu. Bouthier (1993) souligne que les décisions des joueurs ne sont pas que rationnelles au sens où elles sont le fruit de l'interaction des autres composantes de l'action. Bouthier (2000) met aussi en avant le fait que le référentiel commun ne se réduit pas aux aspects décisionnels, mais intègre aussi l'expérience des joueurs dans ses aspects conceptuels certes, mais aussi moteurs, sensibles, et sociaux. L'analyse des situations d'un point de vue externe, est utile pour optimiser les décisions des joueurs, mais il faut savoir aussi la relativiser. En effet, d'une part les modèles de référence sont variés, et l'appropriation de ces modèles est ellemême diversifiée, que ce soit chez les professeurs ou les joueurs.

Une des difficultés de l'enseignement et de l'apprentissage dans les sports collectifs provient de notre difficulté à décrire clairement la nature des apprentissages visés et des dispositifs qui concourent à la réussite dans un environnement très mouvant. Dans le cadre de la formation initiale et continue des maîtres, cette synthèse devrait permettre d'enrichir le cadre de références des enseignants d'EPS non spécialistes des sports collectifs à l'école en vue de nourrir une évaluation de départ et une évaluation formative 


\section{eJRIEPS 30 juillet 2013}

appropriée. Ce type de recueil de données qui permet ensuite de guider les élèves dans leurs apprentissages demande une grande précision sur ce qu'ils savent réellement faire. Nous pensons que les modélisations et les configurations prototypiques présentées devraient aider à la conception des cycles et des leçons de sport collectif.

\section{Bibliographie}

Berchebru, M. (2007). Didactique de l'enseignement des sports collectifs. Quand l'utilisation de technologies multimédia et de la verbalisation améliore l'enseignement des sports collectifs. Mémoire de Mastère (non publié). Université de Franche-Comté

Bourdieu, P. (1980). Le sens pratique. Paris : Les Editions de Minuit.

Bouthier, D. (1986). Comparaison expérimentale des effets de différents modèles didactiques des sports collectifs. In SNEP (Ed.), E.P.S. contenus et didactique (pp. 85-89). Paris : SNEP.

Bouthier, D. (1993). L'approche technologique en S.T.A.P.S. : représentations et actions en didactique des APS. Diplôme d'habilitation à diriger des recherches. Orsay : Université Paris-Sud.

Bouthier, D. (1997). Technicité collective et actions sportives créatrices: rapports contradictoires ou dialectiques ; l'exemple des combinaisons en jeu déployé dans le rugby français. In A. Menaut, Technique et créativité dans les jeux sportifs en Aquitaine, Euskadi, Navarre (pp. 159-173). Bordeaux : Université Bordeaux 2.

Bouthier, D. (2000). La coordination des décisions individuelles ; contribution de l'intelligence tactique. Colloque Préparation Olympique L'évolution de la pensée tactique, Noisy le Grand, 14 et 15 novembre 2000.

Bunker, D., \& Thorpe, R. (1982). A model for the teaching of games in the secondary schools. The Bulletin of physical education, 18, 5-8.

Deleplace, R. (1966). Le rugby. Paris : Colin - Bourrelier.

Deleplace, R. (1979). Rugby de mouvement, rugby total. Paris : Revue EPS.

Caron, J., \& Pelchat, C. (1974). Le Hockey, Sport Collectif : Modèle Empirique ou Théorique. Mouvement, 9, 33-46.

Dewey, J. (1947). Expérience et éducation [Experience and education, 1938], (trad. M.-A. Carroi). Paris : Bourrelier.

Frith, C. (2007). Making up the Mind. How the brain creates our mental world. Oxford: Blackwell Plubishing. 


\section{eJRIEPS 30 juillet 2013}

Gréhaigne, J.F. (1989). Football de mouvement. Vers une approche systémique du jeu.

Thèse de Doctorat (non publiée). Université de Bourgogne, Dijon.

Gréhaigne, J.F. (1992). L'organisation du jeu en football. Paris : ACTIO.

Gréhaigne, J.F. (Ed.). (2007). Configurations du jeu, débat d'idées et apprentissage des sports collectifs. Besançon : Presses de l'Université de Franche-Comté.

Gréhaigne, J.F. (Ed.). (2011). Des signes au sens. Le jeu, les indices, les postures et les apprentissages dans les sports collectifs à l'école. Besançon: Presses de l'Université de Franche-Comté.

Gréhaigne, J.F., \& Godbout, P. (1995). Tactical knowledge in team sports from a constructivist and cognitivist perspective. Quest, 47, 490-505.

Gréhaigne, J.F., \& Godbout, P. (2012). (2012). À propos de la dynamique du jeu ... en football et autres sports collectifs. eJRIEPS, 26, 130-156.

Gréhaigne, J.F., Godbout, P., \& Zerai, Z. (2010). Using complex system analysis to model team ball sports International Journal of Physical Education (4) 31-41.

Gréhaigne. J-F., Zerai, Z., \& Caty, D. (2009). Prototypic Configurations of Play in Handball in Physical Education: A Strategy to Promote Student Understanding in Team Sports. In T. Hopper, J. Butler \&B. Storey (Eds.) TGFU Simply good pedagogy; understanding a complex challenge (pp. 51-62). Physical and Health Education Canada.

Leplat, J. (2000). L'environnement de l'action en situation de travail. In J.M. Barbier (Ed.), L'analyse de la singularité de l'action (pp. 107-132). Paris : PUF.

Mérand, R. (Ed.) (1990). Basket-ball : lancer ou circuler ? Rencontre pédagogiques, 28, 9109.

Mialaret, G. (1998). Savoirs théoriques, savoirs scientifiques et savoirs d'action en éducation. In J.M. Barbier (161-188), Savoirs théoriques et savoirs d'action. Paris : PUF.

Mahlo, F. (1969). Acte tactique en jeu. Paris : Vigot.

Mylopoulos, J. (1992). Conceptual modeling and Telos. In Loucopoulos, P.; Zicari, R. Conceptual Modeling, Databases, and Case An integrated view of information systems development (pp. 49-68). New York: Wiley.

Ochanine, D. (1969). Rôle de l'image opérative dans la saisie du contenu informationnel des signaux. Questions de Psychologie, 4.

Ochanine, D. (1978). Le rôle des images opératives dans la régulation des activités de travail. Psychologie et Education, 2, 63-72. 


\section{eJRIEPS 30 juillet 2013}

Ochanine, D., Quaas, W., \& Zaltzman, A. (1972). Déformation fonctionnelle des images opératives. Questions de Psychologie, 3.

Paillard, J. (1987). Système nerveux et fonctions d'organisation. In J. Piaget, J.P. Bronckart, \& P. Mounoud (Eds.), La Psychologie (pp. 1378-1441).Paris : Gallimard, Encyclopédie de la Pléïade.

Ripoll, H., Zoudji, B., \& Lluccia, L. (2009). Utiliser des images pour entraîner au football. In B. Zoudji (Ed.) Science \& Football, recherches et connaissances actuelles (pp. 355-362). Valenciennes : Presses Universitaires.

Turner, A.P., \& Martinek, T. (1992). A comparative analysis of two models for teaching games (Technique Approach and Game-Centered (Tactical Focus) Approach). International Journal of Physical Education, 29 (4) 15-31.

Villepreux, P. (1987). Rugby de mouvement et disponibilité du joueur. Mémoire INSEP, Paris.Wallon, H. (1941). L'évolution psychologique de l'enfant. Paris : A. Colin.

Zerai, Z. (2009). Origine culturelle et apprentissage des sports collectifs chez les jeunes filles. Étude comparative d'élèves tunisiennes et françaises. Rapport de recherche IUFM de Franche-Comté.

Zerai, Z., Gréhaigne J-F., \& Godbout, P. (2011). Des signes de ressemblance dans les configurations du jeu. In J.F. Gréhaigne (Ed.), Des signes au sens. Le jeu, les indices, les postures et les apprentissages dans les sports collectifs à l'école (pp. 73-84). Besançon : Presses de l'Université de Franche-Comté.

Z. Zerai, Gréhaigne, J-F, \& P. Godbout (2013). Configurations of play in invasion team sports and learning by analogy. International Journal of Physical Education Volume L (1) 18-28. 